\title{
Development of the chloroplast genome-based InDel markers in Niitaka (Pyrus pyrifolia) and its application
}

\author{
Ho Yong Chung ${ }^{1,3}$. So Youn Won ${ }^{1} \cdot$ Yoon-Kyung Kim ${ }^{2}$ Jung Sun Kim
}

Received: 8 November 2018 / Accepted: 25 December 2018 / Published online: 9 January 2019

(c) The Author(s) 2019

\begin{abstract}
Pears (Pyrus spp.) are one of the most important fruit crops in temperate regions and are self-incompatible. Large numbers of interspecific hybrids occur naturally and, have been artificially produced in breeding programs. Also, many pear cultivars were generated from intraspecific crossing. Some varieties have been cultivated for a long time, but their breeding history remains unknown. The $P$. pyrifolia $\mathrm{cv}$. Niitaka is one of key varieties in the Korean pear breeding program. Therefore, its complete chloroplast (cp) genome was determined, and its phylogenetic relationships with other Pyrus species and $P$. pyrifolia cultivars were addressed. The cp genome has a total of 133 genes, including 93 protein-coding genes, 32 tRNA genes, and eight rRNA genes. We found many SNPs in the 'Niitaka' cp genes when compared with that in another Korean cultivar 'Wonwhang' (BioSample SAMN05196235). The primer sets for six genes that had two or more SNPs in their sequence were used to amplify and sequence 27 Pyrus and one Malus cultivars. Of these, we found dramatic InDel polymorphisms in the $n d h A$ and $c l p P$ genes. Phylogenetic relationships using the sequences of these two genes in 28 samples showed that they could mainly be classified into two groups of $P$. pyrifolia. Group I constitutes Niitaka and all cultivars that contain chloroplast maternally inherited from Niitaka, and group II constitutes the other cultivars of $P$. pyrifolia. We have developed a useful polymorphic molecular marker to confirm the maternal parent in the interspecific hybrids of Niitaka and previous mothers of Niitaka, (such as Amanogawa). Furthermore, these two genes could identify and greatly aid in understanding the subspecies classification in Pyrus.
\end{abstract}

Keywords Asian pears $\cdot$ Chloroplast genome $\cdot$ Chloroplast InDel markers $\cdot$ Next-generation sequencing $\cdot$ Phylogenetic analysis

Ho Yong Chung and So Youn Won equally contributed as co-first authors.

Electronic supplementary material The online version of this article (https://doi.org/10.1007/s11816-018-00513-0) contains supplementary material, which is available to authorized users.

Jung Sun Kim

jsnkim@korea.kr

Ho Yong Chung

hychung75@korea.kr

So Youn Won

soyounwon@korea.kr

Yoon-Kyung Kim horti8992@korea.kr

\section{Introduction}

The chloroplast is a photosynthetic organelle used during the biosynthetic pathways of fatty acids, vitamins, pigments and amino acids (Bobik and Burch-Smith 2015; RodríguezEzpeleta et al. 2005). The cp genome among land plants has a highly conserved genome structure with a large single copy region (LSC) and a small single copy region (SSC) separated by two inverted repeat regions (IR) (Chumley et al. 2006;

1 Department of Agricultural Biotechnology, National Institute of Agricultural Sciences, RDA, Jeonju 54874, Republic of Korea

2 Pear Research Institute, National Institute of Horticultural and Herbal Science, RDA, Naju 58216, Republic of Korea

3 Present Address: 3BIGS, B-302, 160, Dongtanbanseok-ro, Hwaseong-si, Gyeonggi-do 18454, Republic of Korea 
Wicke et al. 2011). The complete cp genome sequences from tobacco and liverwort were reported in 1986 (Ohyama et al. 1986; Shinozaki et al. 1986). Since then, the number of fully sequenced cp genome has been increased rapidly by cost-effective next-generation sequencing technique. Over 800 complete cp genome sequences have been deposited in the National Center for Biotechnology Information (NCBI) organelle genome database including 300 entire cp genomes from crop and tree (Daniell et al. 2016). With the highly conserved sequence, compact size, lack of recombination, and maternal inheritance, the cp genomes have been used for generating genetic markers for phylogenetic classification (Hong et al. 2017), and DNA barcoding system for molecular identification (Dong et al. 2012).

Pears (Pyrus spp.), which belong to the family of Rosaceae, have been cultivated in East Asia, Europe, and North America for more than 3000 years, and are among the most important fruit crops in the temperate regions (Bell 1991). Pyrus has been divided into two groups, occidental, and oriental pears, based on their geographic distribution (Rubtsov 1944). According to their usage, Pyrus accessions are also classified into three groups. The first one comprises $P$. brestchenidri, P. pyrifolia, and P. ussuriensis which are commercially cultivated in Asia. And also, there is European pear (P. communis), the other rootstock group contains $P$. betulaefolia and P. calleryana (Yeo and Reed 1995). However, all species of Pyrus are likely to originate from natural interspecific hybridization events, which make it difficult to determine their exact genetic relationships. Understanding the domestication process of the cultivated pear and the evolutionary process of the pear species will be helpful in exploiting elite genetic resources in pears and aid in modern breeding.

In Korea, pear breeding began in the late 1920s at the National Institute of Horticultural and Herbal Science (NIHHS) of the Rural Development Administration (RDA). Asian pears have a sweet flavor, rich juice, and crisp flesh. Furthermore, they are considered to be of superior quality and more storage friendly compared to Occidental pears (Kim 2016). To improve fruit quality regarding fruit size, sugar content, flesh firmness, and storage quality, many breeders frequently used interspecific hybridization (Iketani et al. 2012). Consequently, the classification of pears is complicated because of the occurrence of both natural and artificial interspecific hybrids. Therefore, the genetic relationships and evolutionary history of Asian pears are unclear (Jiang et al. 2016). Studying the cp genome, which is maternally inherited, will enable us to gain a better understanding of the genetic relationships and evolutionary history of Asian pears (Zhang 2010).

In our previous study, the complete $\mathrm{cp}$ and mitochondrial (mt) genome of a Korean pear (P. pyrifolia cv., Wonhwang), was de novo assembled using whole genome-sequencing data (Chung et al. 2017a, b). In this study, we sequenced the complete cp genome of another Korean variety (P. pyrifolia cv. Niitaka), and identified the deleted sequences in two cp genes of Niitaka and the maternal parent of the interspecific hybrids of Niitaka, despite the fact that species from the same genus of $P$. pyrifolia did not have these deletions. Many studies have employed the comparative genomic analysis using tandem repeats, InDels, simple sequence repeats (SSRs) polymorphism, and genetic diversity to identify valuable markers for DNA barcoding and phylogenetic analysis between species levels. Indel polymorphic markers within species in cp genome are rare, because they occur at a low evolutionary rate in most taxa. We reported the InDel polymorphisms within the $n d h A$ and $c l p P$ gene as different species of Pyrus as well as within these genes in P. pyrifolia.

\section{Materials and methods}

\section{Plant materials and DNA sample preparation}

A total of 27 individuals in the Pyrus genus were cultivated in the Pear Research Station, NIHHS, RDA, Republic of Korea (latitude $35^{\circ} 01^{\prime} 25.6^{\prime \prime} \mathrm{N}$, longitude $126^{\circ} 44^{\prime} 38.4^{\prime \prime} \mathrm{E}$ ). The 16 cultivars of the $P$. pyrifolia, Niitaka, Whangkeumbae, Amanogawa, Gamro, Sunwhang, Shinhwa, Youngsanbae, Hanareum, Wonwhang, Imamuraaki, Geumchonjosaeng, Chuwangbae, Okusankichi, Seolwon, SuperGold, and Chanxixueli and two cultivars of P. ussuriensis, Doonggeullebae, and Cheongdangrori were used. Additionally, Yali and Dangshansuli of $P$. bretschneideri were used. We also used Danbae cultivar, an interspecific cross between $P$. pyrifolia and $P$. communis. As classifying by rootstock group, OPR125 and OPR195 of $P$. calleryana were included. One unclassified pear cultivar used with Kozo and a $P$. faurie cultivar Godang 5-1 were also used. We collected on two European pear cultivars of Bartlett and Max Red Bartlett. As an outgroup sequence cultivar with Malus, Fuji is listed in this analysis. These characteristics of 28 cultivars are listed in Supplement Table 1. Genomic DNA was extracted from young leaves using a DNeasy Plant Mini kit (Qiagen, CA, USA) according to the manufacturer's instructions.

\section{Chloroplast genome sequencing, assembly, and annotation}

Whole genome sequencing was performed using an Illumina genome analyzer (Hiseq4000, Illumina, USA) at Macrogen (http://www.macrogen.com/) in Seoul, Republic of Korea. Genomic libraries with 350-bp inserts were prepared by following the paired-end standard protocol recommended by the manufacturer. Each sample was tagged separately with a different index. De novo assembly was performed using a 
CLC genome assembler (4.06 beta, CLC Inc., Aarhus, Denmark) with parameters of a minimum 200-600 bp autonomously controlled overlap size. The cp-coding contigs were identified by comparison to the entire cp genome of the reported $P$. pyrifolia (Terakami et al. 2012) and circularized. Gene annotation was conducted using CpGAVAS (http:// www.herbalgenomics.org) and the cp map was drawn with the OrganellarGenomeDRAW (OGDRAW) program (https ://chlorobox.mpimp-golm.mpg.de/OGDraw.html).

\section{Development of chloroplast InDel markers}

The chloroplast sequence comparison of Niitaka vs. Wonwhang revealed many SNPs. From them, we selected six protein-coding genes that had more than two SNPs in this comparison. They are the ATP synthase subunit beta (atpB), ATP-dependent Clp protease ( $c l p P)$, NADH dehydrogenase subunit $5(n d h F)$, NADH dehydrogenase subunit 4 (ndhD), NADH dehydrogenase subunit 1 (ndhA), and yeast cadmium factor $1(y c f l)$ genes. The primer set was designed using the Primer 3 program (http://bioinfo.ut.ee/primer3-0.4.0/). Six primers paired sequences were designed including the two SNPs with amplicon size of approximately $1 \mathrm{~kb}$. Based on chloroplast genome (NC_015996 and KX904342), the polymorphic gene name, SNP location, primer pair sequences, annealing temperature, and expected product size are described in Table 1. PCR amplifications were carried out in $30 \mu \mathrm{l}$ reaction containing $30 \mathrm{ng}$ of genomic DNA template, $15 \mu \mathrm{l}$ of $2 \mathrm{X}$ TOPsimpleTM DyeMIX-nTaq (Enzynomics, Korea), and $10 \mathrm{pM}$ each of forward and reverse primers. The amplification was conducted by PCR machine (AB GeneAmp PCR system 9700) with a hot start of $94{ }^{\circ} \mathrm{C}$ for $5 \mathrm{~min}, 35$ cycles of $30 \mathrm{~s}$ at $94^{\circ} \mathrm{C}, 30 \mathrm{~s}$ at $60^{\circ} \mathrm{C}$, and $1 \mathrm{~min}$ at $72{ }^{\circ} \mathrm{C}$, and a final extension at $72{ }^{\circ} \mathrm{C}$ for $10 \mathrm{~min}$. Amplified fragments were analyzed by separation on agarose gels and sequencing. Then, the sequences were aligned with DNAstar software (DNASTAR Inc., Madison, Wisconsin USA) to compare each sequence.

\section{Phylogenetic analysis}

Phylogenetic analysis was conducted using the two InDel marker regions, $n d h A$, and $c l p P$, shared by 27 Pyrus and one Malus. These Sanger sequences are shown in supplement file 5. Multiple sequence alignments were carried out using ClustalW, which was followed by phylogenetic tree generation using MEGA7 (Kumar et al. 2016) with 1000 bootstrap replicates. $M$. pumila $\mathrm{cv}$. Fuji used as an outgroup. The maximum likelihood method was used, and the tree with the highest log likelihood was obtained.

\section{Results}

\section{The complete chloroplast genome of Niitaka}

We sequenced, and de novo assembled the cp genome of $P$. pyrifolia cv. Niitaka. The entire cp genome sequences of Niitaka was submitted to GenBank under the accession number KX904342. The complete cp genome of Niitaka is $159,922 \mathrm{bp}$ in length, with a pair of IR regions of 26,392 bp that separate an LSC region of 72,023 bp and an SSC region of $19,235 \mathrm{bp}$. A total of 133 genes were identified including 93 protein-coding genes, 32 tRNA genes, and 8 rRNA genes (Fig. 1). Twelve genes, atpF, ndhA, two $n d h B$, two rpl2, rpoC1, rpl22, two trnI-TAT and two ycf15 have one intron, and $c l p P$ and $y c f 3$ gene have two introns. The SSC region contains 11 protein-coding, and 1 tRNA genes, and the respective IR regions contain 9 protein-coding genes, 5 tRNAs, and 4 rRNAs.
Table 1 Information on primers for amplifying the six genes from Pyrus, including Malus

\begin{tabular}{|c|c|c|c|c|c|}
\hline Target gene & $\begin{array}{l}\text { CP genome } \\
\text { location }\end{array}$ & SNP & Sequence $\left(5^{\prime}-3^{\prime}\right)$ & $T_{\mathrm{m}}\left({ }^{\circ} \mathrm{C}\right)$ & Product size (bp) \\
\hline \multirow[t]{2}{*}{ atpB } & 56843 & $\mathrm{~A} \rightarrow \mathrm{G}$ & \multirow{2}{*}{$\begin{array}{l}\text { ATACCCTTGGCAGCTAATCC } \\
\text { CAACGCTCTGGTAGTTAGGG }\end{array}$} & 58 & \multirow[t]{2}{*}{951} \\
\hline & 57574 & $\mathrm{G} \rightarrow \mathrm{T}$ & & 58 & \\
\hline \multirow[t]{2}{*}{ clpP } & 73810 & $\mathrm{~A} \rightarrow \mathrm{T}$ & TAATGGGCTGGTTTAGATCG & 58 & \multirow[t]{2}{*}{990} \\
\hline & 73811 & $\mathrm{~A} \rightarrow \mathrm{C}$ & CTATGCCTTCGCGCTATATG & 59 & \\
\hline \multirow[t]{2}{*}{$n d h F$} & 114776 & $\mathrm{G} \rightarrow \mathrm{A}$ & CGACTCCATTTGTAATTCCATC & 58 & \multirow[t]{2}{*}{906} \\
\hline & 114872 & $\mathrm{~T} \rightarrow \mathrm{C}$ & CCCTTGCTTGTTTTTGGTC & 58 & \\
\hline \multirow[t]{2}{*}{$n d h D$} & 121354 & $\mathrm{G} \rightarrow \mathrm{T}$ & AAGATTCCGGCTAAAAGCAT & 58 & \multirow[t]{2}{*}{1009} \\
\hline & 121871 & $\mathrm{~T} \rightarrow \mathrm{C}$ & TTCTGCTCCAAGAACAGAGG & 58 & \\
\hline \multirow[t]{2}{*}{$n d h A$} & 125948 & $\mathrm{~A} \rightarrow \mathrm{C}$ & TAGGGACCGCGTCAGTATTC & 58 & \multirow[t]{2}{*}{1066} \\
\hline & 126257 & $\mathrm{~T} \rightarrow \mathrm{A}$ & TCTCTACGTGCGATTCGTTG & 58 & \\
\hline \multirow[t]{2}{*}{$y c f 1$} & 130405 & $\mathrm{~A} \rightarrow \mathrm{T}$ & CAATATTGATCCAGGCCTCA & 58 & \multirow[t]{2}{*}{990} \\
\hline & 130846 & $\mathrm{G} \rightarrow \mathrm{C}$ & AAAAAGAAGAACTGCTCGATT & 56 & \\
\hline
\end{tabular}






Fig. 1 Gene map of the Niitaka chloroplast genome. The quadripartite structure includes two copies of an IR region (IRA and IRB) that separate the large single copy (LSC) and small single copy (SSC) regions. Genes on the outside of the map are transcribed in the coun-

\section{Chloroplast InDel markers development}

We determined the polymorphisms in the cp genomes between Niitaka and one of Asian pear cultivar with early ripening traits, 'Wonwhang' (Chung et al. 2017a, b). The sequence comparison of the two cp genomes identified many SNPs between Niitaka and Wonwhang. In particular, we found the 29 SNPs in 20 protein-coding genes ter-clockwise direction and genes on the inside of the map are transcribed in the clockwise direction. Differential functional gene groups are color-coded. The dashed area in the inner circle indicates the GC content of the chloroplast genome that belonged to the diverse functional groups including house-keeping function and photosynthesis (Supplement Table 2). While most of the polymorphic genes had a single SNP, atpB for ATP synthase, three NADH dehydrogenase genes ( $n d h A, n d h D$, and $n d h F), p s a A$ for photosystem I, and a hypothetical protein $y c f 1$ had two SNPs (Supplement Table 2). ATP-dependent protease $C l p P$ contained as many as four SNPs (Supplement Table 2). However, 
we did not observe any InDel between two cultivars in the protein-coding genes.

Next, we determined polymorphisms among Pyrus species. For this, the PCR primers were designed from six genes that showed two or more SNPs above (Table 1). The PCR and the subsequent Sanger sequencing with four proteincoding genes, $n d h D, n d h F, y c f 1$, and $a t p B$ discovered SNPs but not Indels among 27 Pyrus and one Malus individuals (Supplement Table 2). However, two genes $n d h A$ and $c l p P$ had both types of polymorphisms, SNPs and InDels. The nucleotide (nt) length of the $n d h A$ amplicon ranged from 957 to $980 \mathrm{bp}$, and nt length of $c l p P$ amplicon ranged from 933 to $958 \mathrm{bp}$. The length of the InDel regions in $n d h A$, and $c l p P$ were $23 \mathrm{bp}$ with '5-ATTAAGGATTAAAGTAAC

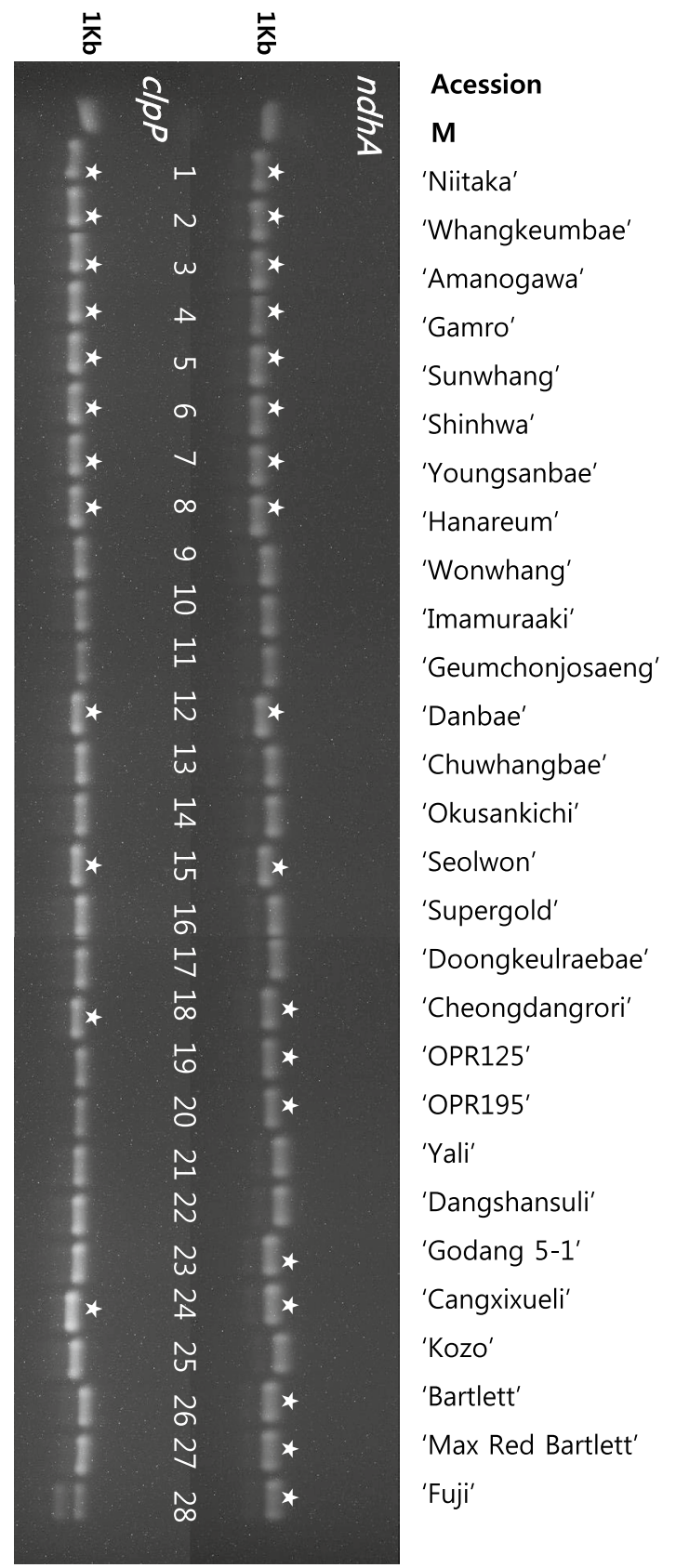

Fig. 2 Agarose gel electrophoresis of the amplicons of the $n d h A$ and clpP chloroplast gene-specific primers (left photo). These chloroplast InDel markers were found in the 27 Pyrus and one Malus cultivars. M: DNA size marker (Enzynomics DM003, $10 \mu \mathrm{l}$ of $150 \mu \mathrm{g} / \mathrm{ml}$ ). The electrophoresis was performed on a $2.5 \%(n d h A)$ and $3 \%(c l p P)$ aga-

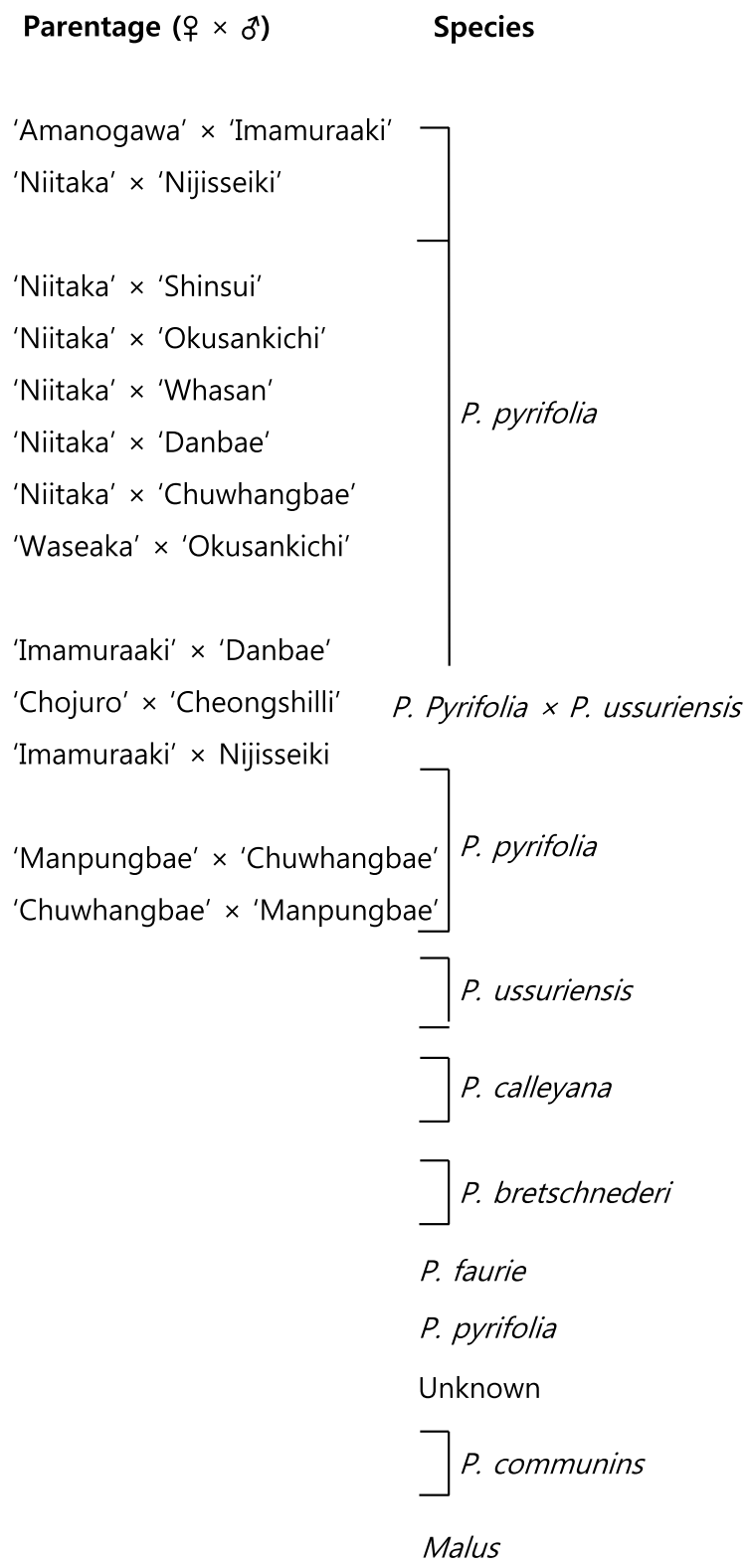

rose gel and run for $8 \mathrm{~h}$ at $100 \mathrm{~V}(n d h A)$ and $16 \mathrm{~h}$ at $80 \mathrm{~V}(\mathrm{clpP})$. The amplified products with indels were represented with asterisk, respectively. We assigned the accessions, intercross parentage, and species to each lane number 
AAAGA-3' and 10 bp with '5-T...CAAGATTTT-3', respectively (Fig. 2). $C l p P$ gene are located in $73,417-75,471 \mathrm{bp}$ and $n d h A$ gene which corresponds to $124,649-126,895 \mathrm{bp}$. $\mathrm{ClpP}$ gene has three exons and two introns and all of SNPs and InDel sequences from these two intron regions in our experiment. We found two SNPs of $c l p P$ gene in the first intron with 73,810 and 73,811 bp (Table 1) and this could be detected in variable deletions based on 11 bp less and more in the second intron (Supplement Figure 3). NdhA gene has two exons and an intron and SNPs and Indel was detected from an intron region (Supplement Figure 4). Deleted sequences were the same in the maternal parent of the interspecific hybrids of Niitaka and previous mothers of Niitaka, such as Amanogawa, as shown in Fig. 2, lanes 1-8. Danbae (lane 12), Seolwon (lane 15), Cheongdangrori (lane 18) and Chanxixueli (lane 24) had the same deleted sequences in this study. However P. calleryana, OPR125 and OPR195 (lanes 19 and 2), P. faurie, Godang 5-1 (lane 23), P. communis, Bartlett and Max Red Bartlett (lanes 26 and 27), and Malus, Fuji (lane 28) did not have a deleted sequences of
clpP gene. The comparative SNPs and InDel sequences of $n d h A$ and $c l p P$ amplicons in 28 cultivars from 166 to $230 \mathrm{bp}$ and from 486 to $530 \mathrm{bp}$, respectively, are summarized in Tables 2 and 3.

\section{Phylogenetic tree}

Phylogenetic trees were constructed by conducting maximum likelihood analysis with MEGA7 program and each $n d h A$ and $c l p P$ amplicon sequences of 27 Pyrus with Malus being as the outgroup (Figs. 3, 4). It was found that the occidental pears (Bartlett and max red Bartlett) were located outside of the oriental groups. The P. faurie cv. Godang 5-1 was located between the Asian and European pears. Whangkeumbae, Gamro, Sunwhang, Sinhwa, Youngsanbae, and Hanareum contained maternally inherited chloroplast from Niitaka according to the Korean Pear Breeding Program. Considering that Niitaka was generated from the crossing between Amanogawa (mother) and Imamuraaki (father) (Kim and Nou 2016). Geumchonjosaeng, Chuwhangbae,

Table 2 The comparative SNPs and InDel sequences of $n d h A$ gene amplicons in 27 Pyrus and one Malus

\begin{tabular}{|c|c|c|}
\hline No. & Variety & InDel region sequences in intron of $n d h A$ gene \\
\hline 1 & Niitaka & 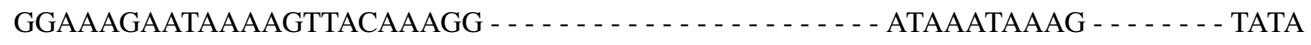 \\
\hline 2 & Whangkeumbae & GGAAAGAATAAAAGTTACAAAGG - - - - - - - - - - - - ATAAATAAAG - - - - - TATA \\
\hline 3 & Amanogawa & GGAAAGAATAAAAGTTACAAAGG - - - - - - - - - - - - ATAAATAAAG - - - - - TATA \\
\hline 4 & Gamro &  \\
\hline 5 & Sunwhang & 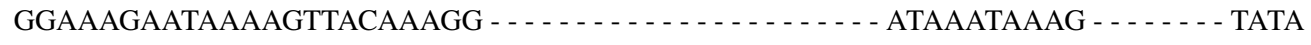 \\
\hline 6 & Shinhwa & 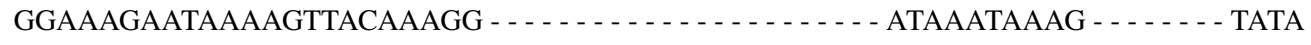 \\
\hline 7 & Youngsanbae & GGAAAGAATAAAAGTTACAAAGG - - - - - - - - - - - - - ATAAATAAAG - - - - - TATA \\
\hline 8 & Hanareum & GGAAAGAATAAAAGTTACAAAGG $-\cdots$ \\
\hline 9 & Wonwhang & GGAAAGAATAAAAGTAACAAAGGTTGGATTAAAGTAACAAAGAATAAATAAAG - - - - - - - TATA \\
\hline 10 & Imamuraaki & GGAAAGAATAAAAGTAACAAAGGTTGGATTAAAGTAACAAAGAATAAATAAAG - - - - - - - TATA \\
\hline 11 & Geumchonjosaeng & GGAAAGAATAAAAGTAACAAAGGTTGGATTAAAGTAACAAAGAATAAATAAAG - - - - - - - TATA \\
\hline 12 & Danbae & GGAAAGAATAAAAGTTACAAAGG - - - - - - - - - - - - - ATAAATAAAG - - - - - TATA \\
\hline 13 & Chuwhangbae & GGAAAGAATAAAAGTAACAAAGGTTGGATTAAAGTAACAAAGAATAAATAAAG - - - - - - - TATA \\
\hline 14 & Okusankichi & GGAAAGAATAAAAGTAACAAAGGTTGGATTAAAGTAACAAAGAATAAATAAAG - - - - - - TATA \\
\hline 15 & Seolwon & GGAAAGAATAAAAGTTACAAAGG $-\cdots$ \\
\hline 16 & Supergold & GGAAAGAATAAAAGTAACAAAGGTTGGATTAAAGTAACAAAGAATAAATAAAG - - - - - - - TATA \\
\hline 17 & Doonggeullebae & GGAAAGAATAAAAGTAACAAAGGTTGGATTAAAGTAACAAAGAATAAATAAAG - - - - - - - TATA \\
\hline 18 & Cheongdangrori & GGAAAGAATTAAAGTTACAAAGG - - - - - - - - - - - - - - ATAAATAAAG - - - - - - TATA \\
\hline 19 & OPR125 & GGAAAGAATAAAAGTAACAAAGG - - - - - - - - - - - ATAAATAAAG - - - - - TATA \\
\hline 20 & OPR195 & GGAAAGAATAAAAGTAACAAAGG $-\cdots$ \\
\hline 21 & Yali & GGAAAGAATTAAAGTAACAAAGGTTGGATTAAAGTAACAAAGAATAAATAAAG - - - - - - TATA \\
\hline 22 & Dangshansuli & GGAAAGAATTAAAGTAACAAAGGTTGGATTAAAGTAACAAAGAATAAATAAAG - - - - - - TATA \\
\hline 23 & Godang 5-1 & GGAAAGAATTAAAGTAACAAAGG - - - - - - - - - - - - - - ATAAATAAAG - - - - - TATA \\
\hline 24 & Cangxixueli & GGAAAGAATTAAAGTAACCAAGG $-\cdots$ \\
\hline 25 & Kozo & GGAAAGAATAAAAGTAACAAAGGTTGGATTAAAGTAACAAAGAATAAATAAAG - - - - - - TATA \\
\hline 26 & Bartlett & GGAAAGAATTAAAGTTACCAAGG - - - - - - - - - - - ATAAATAAAG $-\cdots$ \\
\hline 27 & Max red bartlett & GGAAAGAATTAAAGTTACCAAGG $-\cdots$ \\
\hline 28 & Fuji & GGGAAGGATTAAAGTTACACATT - - - - - - - - - - - - - - - - TTAAAAAAAAGAAATTTTTTTT \\
\hline
\end{tabular}


Table 3 The comparative SNPs and InDel sequences of clpP gene amplicons in 27 Pyrus and one Malus

\begin{tabular}{|c|c|c|}
\hline No. & Variety & InDel region sequences in the second intron of $c l p P$ gene \\
\hline 1 & Niitaka & TCCCAAAGTTC - - - - TTTTTTTTTTCAAATAAAAAAA - - - - - - - - TTTTATTTTCATATTCTT \\
\hline 2 & Whangkeumbae & TCCCAAAGTTC - - - - TTTTTTTTTTCAAATAAAAAAA - - - - - - - - TTTTATTTTCATATTCTT \\
\hline 3 & Amanogawa & TCCCAAAGTTC - - - TTTTTTTTTTCAAATAAAAAAA - - - - - - - TTTTATTTTCATATTCTT \\
\hline 4 & Gamro & TCCCAAAGTTC - - - - TTTTTTTTTTCAAATAAAAAAA - - - - - - - TTTTATTTTCATATTCTT \\
\hline 5 & Sunwhang & TCCCAAAGTTC - - - - TTTTTTTTTTCAAATAAAAAAA - - - - - - - TTTTATTTTCATATTCTT \\
\hline 6 & Shinhwa & TCCCAAAGTTC - - - - TTTTTTTTTTCAAATAAAAAAA - - - - - - - - TTTTATTTTCATATTCTT \\
\hline 7 & Youngsanbae & TCCCAAAGTTC - - - - TTTTTTTTTTCAAATAAAAAAA - - - - - - - - TTTTATTTTCATATTCTT \\
\hline 8 & Hanareum & TCCCAAAGTTC - - - - TTTTTTTTTTCAAATAAAAAAA - - - - - - - - TTTTATTTTCATATTCTT \\
\hline 9 & Wonwhang & TCCCAAAGTTC - - TTTTTTTTTTTCAAATAAAAAAACAAGATTTTTTTTATTTTCATATTCTT \\
\hline 10 & Imamuraaki & TCCCAAAGTTC - - - TTTTTTTTTTTCAAATAAAAAAACAAGATTTTTTTTATTTTCATATTCTT \\
\hline 11 & Geumchonjosaeng & TCCCAAAGTTC - - - TTTTTTTTTTTCAAATAAAAAAACAAGATTTTTTTTATTTTCATATTCTT \\
\hline 12 & Danbae & TCCCAAAGTTC - - - - TTTTTTTTTTCAAATAAAAAAA - - - - - - - - TTTTATTTTCATATTCTT \\
\hline 13 & Chuwhangbae & TCCCAAAGTTC - - - TTTTTTTTTTTCAAATAAAAAAACAAGATTTTTTTTATTTTCATATTCTT \\
\hline 14 & Okusankichi & TCCCAAAGTTC - - - TTTTTTTTTTTCAAATAAAAAAACAAGATTTTTTTTATTTTCATATTCTT \\
\hline 15 & Seolwon & TCCCAAAGTTC - - - - TTTTTTTTTTCAAATAAAAAAA - - - - - - - TTTTATTTTCATATTCTT \\
\hline 16 & Supergold & TCCCAAAGTTC - - - TTTTTTTTTTTCAAATAAAAAAACAAGATTTTTTTTATTTTCATATTCTT \\
\hline 17 & Doonggeullebae & TCCCAAAGTTC - - - TTTTTTTTTTTCAAATAAAAAAACAAGATTTTTTTTATTTTCATATTCTT \\
\hline 18 & Cheongdangrori & TCCCAAAGTTC - - - - TTTTTTTTTTCAAATAAAAAAA - - - - - - - - TTTTATTTTCATATTCTT \\
\hline 19 & OPR125 & TCCCAAAGTTC - - - TTTTTTTTTTTCAAATAAAAAAACAAGATTTTTTTTATTTTCATATTCTT \\
\hline 20 & OPR195 & TCCCAAAGTTC - - - TTTTTTTTTTTCAAATAAAAAAACAAGATTTTTTTTATTTTCATATTCTT \\
\hline 21 & Yali & TCCCAAAGTTC - - - TTTTTTTTTTTCAAATAAAAAAACAAGATTTTTTTTATTTTCATATTCTT \\
\hline 22 & Dangshansuli & TCCCAAAGTTC - - - TTTTTTTTTTTCAAATAAAAAAACAAGATTTTTTTTATTTTCATATTCTT \\
\hline 23 & Godang 5-1 & TCCCAAAGTTC - TTTTTTTTTTTTTCAAATAAAAAAACAAGATTTTTTTTATTTTCATATTCTT \\
\hline 24 & Cangxixueli & TCCCAAAGTTC - - - - TTTTTTTTTTCAAATAAAAAAA - - - - - - - TTTTATTTTCATATTCTT \\
\hline 25 & Kozo & TCCCAAAGTTC - - - TTTTTTTTTTTCAAATAAAAAAACAAGATTTTTTTTATTTTCATATTCTT \\
\hline 26 & Bartlett & TCCCAAAGTTCTTTTTTTTTTTTTTCAAATAAAAAAACAAGATTTTTTTTATTTTCATATTCTT \\
\hline 27 & Max red bartlett & TCCCAAAGTTCTTTTTTTTTTTTTTCAAATAAAAAAACAAGATTTTTTTTATTTTCATATTCTT \\
\hline 28 & Fuji & TCCСAAAGTTCTTTTTTTTTTTTTTCAAATAAAAAAACAAGATTTTTTTTATTTTCATATTCTT \\
\hline
\end{tabular}

and SuperGold were generated as maternally inherited chloroplast from Imamuraaki. Most of Pyrus genus come together with the same subgroups, however, two $P$. ussuriensis cultivars, Cheongdangrori and Doonggeullebae were divided into the other groups.

\section{Discussion}

Chloroplast sequences evolve relatively slowly and are highly conserved between species within a genus (Dong et al. 2014). Therefore, chloroplast sequences are ideal for species identification, phylogenetic research, and genetic diversity research, etc. With the decreasing cost of nextgeneration sequencing, sequencing of cp genome and development of cp-based DNA markers have attracted attention (Davey et al. 2011). In this study, we utilized Illumina sequencing to provide the genomic resource and ultimately the whole cp sequences of an Asian pear cultivar, from which we have expected to develop cp-based molecular markers. The obtained cp genome showed the canonical feature of cp genome: the size of higher plants ranging from 120 to $160 \mathrm{~kb}$ and a quadripartite structure consisted of a pair of inverted repeats (IRs), LSC and SSC regions.

While most of sequences were conserved, we found many SNPs in the protein-coding genes from the cp sequences comparison between the two cultivars, Niitaka and Wonwhang, which served as a fundamental resource for marker development, species identification, phylogenetic study, etc. Within the cp genome, the matK, rbcL, rpoB, rpoCl and $y c f l$ genes are the main DNA makers used in identifying genetic diversity in land plants (Dong et al. 2015; Group et al. 2009). In this study, we also observed that matK and $y c f 1$ was polymorphic (Supplement Table 2). Besides, $n d h A$ and $c l p P$ genes were identified to be highly polymorphic and could be used for marker development. SNPs, as well as InDels, were discovered in $n d h A$ and $c l p P$ genes at the level of Pyrus species and cultivars. Recently, InDel markers in cp have been used for genetic identification in many crops such as potato, quinoa, ginseng, onion, and soybean (Hong 
Fig. 3 Maximum likelihood phylogenetic tree of 27 Pyrus species based on chloroplast InDel marker $n d h A$ gene sequences. The numbers on the branches indicate the bootstrap values from 1000 replicates.

Fuji was used as an outgroup

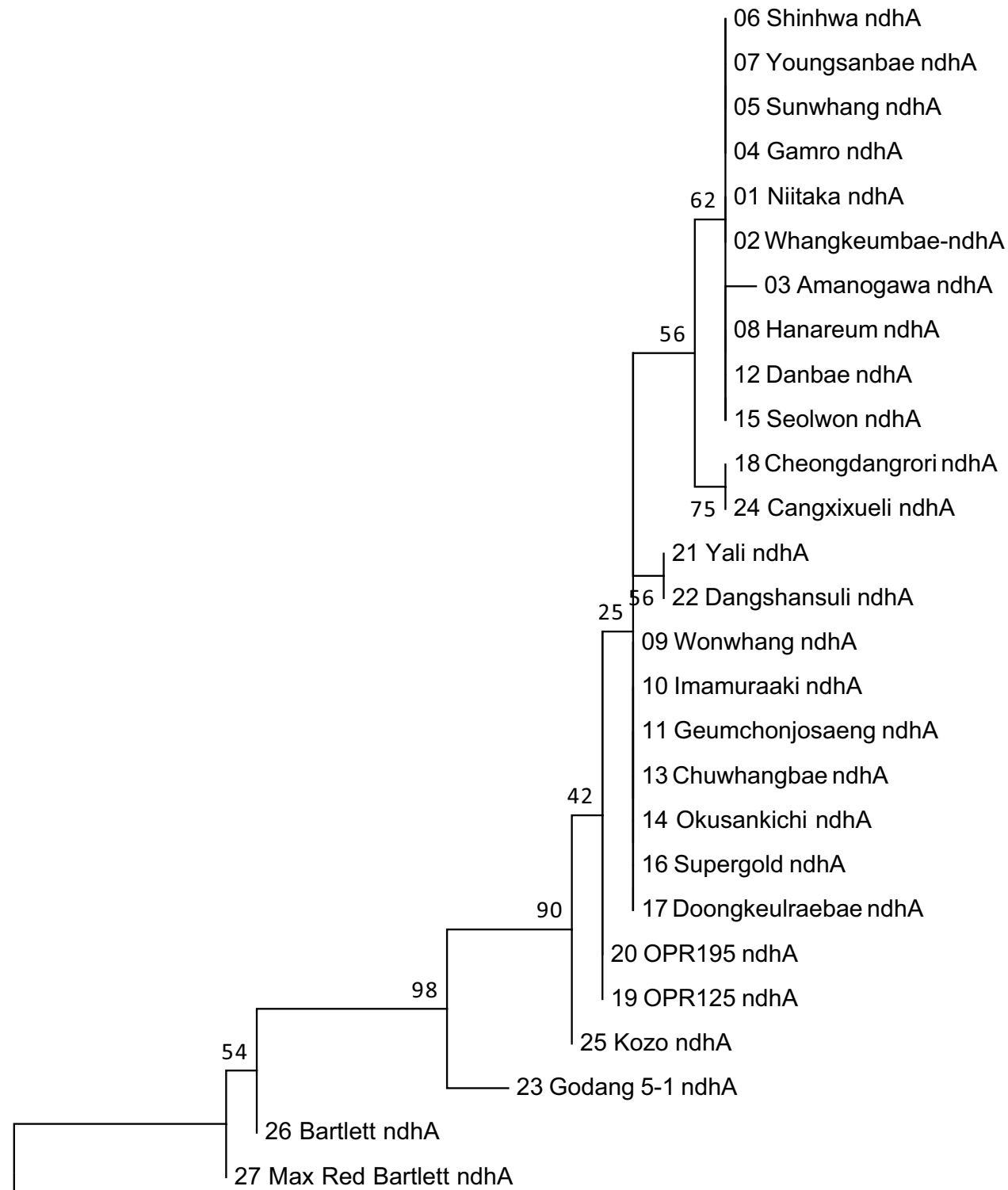

28 Fuji ndhA

0.005

et al. 2017; Joh et al. 2017; Lee et al. 2017; Sohn et al. 2017; Cho et al. 2016). It is easy and inexpensive to use (Qiao et al. 2016). Our primer sets designed for two genes could easily detect InDels among subspecies of $P$. pyrifolia or species of Pyrus on the agarose gel (Fig. 2). Niitaka is an intercross cultivar of Amanogawa and Imamuraaki and is also used as maternal plants for Whankeumbae, Gamro, Sunwhang, Youngsanbae, and Hanareum. The breeding history of Niitaka-related plants was consistent with the genotyping result with the primer pairs for $n d h A$ and $c l p P$ genes, as revealed by the same deletion events of $23 \mathrm{bp}$ as $n d h A$ (5'-ATTAAG GATTAAAGTAACAAAGA-3') and $10 \mathrm{bp}$ as $c l p P\left(5^{\prime}-\mathrm{T} \ldots\right.$
CAAGATTTT-3') (Fig. 2). Danbae, Seolwon, and Cangxixuel of $P$. pyrifolia, Cheongdangrori of $P$. ussuriensis had the $23 \mathrm{bp}$, and $11 \mathrm{bp}$ deleted sequences in their amplicons of $n d h A$ and $c l p P$, respectively. OPR125 and OPR195 of $P$. calleryana, Godang 5-1 of P. faurie, and Bartlett and Max red Bartlett of $P$. communis had the deletion in $n d h A$ but not in $c l p P$. The species $P$. pyrifolia and $P$. ussuriensis did not share the deleted sequences with similar species, $P$. calleryana, $P$. faurie, and $P$. communis, which had a different InDel. These $n d h A$ and $c l p P$ genes could assist in classifying species and even subspecies. 
Fig. 4 Maximum likelihood phylogenetic tree of 27 Pyrus species based on chloroplast InDel marker $c l p P$ gene sequences. The numbers on the branches indicate the bootstrap values from 1000 replicates.

Fuji was used as an outgroup



28 Fuji clpP
The genetic relationships among the Pyrus species were identified. As expected, the European pears were located outside of the Asian pears. P. faurie was present between the Asian and European pears. Unclassified of Pyrus cultivar, Kozo was relevant to $P$. calleryana, $P$. bretschneideri and P.pyrifolia as maternally inherited chloroplast from Imamuraaki. We could confirm that it contained deleted sequences or not in these cultivars, and the deletion of $n d h A$ gene occurred first, followed by deletion of $c l p P$ gene. A molecular population genetic analysis was previously performed with Japanese population of $P$. ussuriensis, and of cultivated pears. They cited the relationships among cultivars and wild populations and used true natives of $P$. ussuriensis that originated in Japan or on the Asian continent (Iketani et al. 2012). In our study, two P. ussuriensis cultivars, Cheongdangrori had the deletion of two genes together, but Doonggeullebae did not have the deletion. Cheongdangrori and Doonggeullebae were showed the different sub-group as phylogenetic relationship. Cheongdangrori belonged to maternally inherited chloroplast from Niitaka, while Doonggeullebae clustered into the other cultivars of $P$. pyrifolia, which included $P$. calleryana, and $P$. bretschneideri. The polymorphisms used for phylogenetic analysis were found in the short sequences of two cp genes, which was not enough to fully distinguish the species. With the completion of genomic sequencing and cp-genome assembly, the 
phylogenetic analysis will be repeated to confirm this present result and further understand the genetic relationship with the higher resolution.

Maternal origins of the Asian pears are still unclear because of frequent hybrid events, fast radial evolution, and lack of informative data. Pyrus pyrifolia cv. Niitaka is the leading cultivar accounting for over $80 \%$ of pear production in the Republic of Korea and has been used as a maternal source to develop new cultivars such as Sinhwa, Whangkeumbae, Sunwhang, Hanareum, and Youngsanbae. This study confirmed the breeding history of Niitaka-related plants as well as another important cultivar Imamuraakirelated group. Moreover, Danbae and Seolwon seemed to be generated from the Niitaka, but not their originally recorded maternal plant, and manpungbae, respectively. Admittedly, our results need to be confirmed with more genetic resources and molecular markers later.

The cp genomes are maternally inherited and have markers that are highly conserved within species, i.e., they show a lack of polymorphism. Here, we reported useful polymorphic genetic markers that can be used to examine plant genome evolution and differentiation in Rosaceae.

Acknowledgements This work was carried out with the support of the Cooperative Research Program for Agriculture Science and Technology Development (PJ0133812018, PJ0104502017).

Author contributions JSK conceived and designed all experiments. JSK performed the experiments. HYC and SYW analyzed the data and wrote the paper. Y-KK contributed the materials. SYW and JSK revised the manuscript. All authors read and approved the final manuscript.

\section{Compliance with ethical standards}

Conflict of interest The authors declare that have no conflict of interest.

Ethical standards The authors declare that the experiments comply with current laws of the countries in which the experiments were performed.

Data archiving statement The chloroplast genome sequences of Pyrus has been deposited in the National Center Biotechnology Information (NCBI) under the accession number K904342 (Pyrus pyrifolia_cv. Niitaka), KX450876 (Pyrus pyrifolia_cv. Wonwhang), KX450877 (Pyrus pyrifolia_cv. Whangkeumbae), KX450884 (intercross cultivar of Pyrus pyrifolia and $P$. communis, Greensis), KX450880 (P. bretschneideri cv. Dangshansuli), KX450881 (P. bretschneideri cv. Yali), KX450883 (P. ussuriensis cv. Cheongshilli), and KX450882 (P. ussuriensis cv. Xioxiangshui).

OpenAccess This article is distributed under the terms of the Creative Commons Attribution 4.0 International License (http://creativeco mmons.org/licenses/by/4.0/), which permits unrestricted use, distribution, and reproduction in any medium, provided you give appropriate credit to the original author(s) and the source, provide a link to the Creative Commons license, and indicate if changes were made.

\section{References}

Bell RL (1991) Pears (Pyrus). Acta Hortic 290:657-700

Bobik K, Burch-Smith TM (2015) Chloroplast signaling within, between and beyond cells. Front Plant Sci 6:781

Cho K-S, Cheon K-S, Hong S-Y, Cho J-H, Im J-S, Mekapogu M, Yu Y-S, Park T-H (2016) Complete chloroplast genome sequences of Solanum commersonii and its application to chloroplast genotype in somatic hybrids with Solanum tuberosum. Plant Cell Rep 35(10):2113-2123

Chumley TW, Palmer JD, Mower JP, Fourcade HM, Calie PJ, Boore JL, Jansen RK (2006) The complete chloroplast genome sequence of Pelargonium $\times$ hortorum: organization and evolution of the largest and most highly rearranged chloroplast genome of land plants. Mol Biol Evol 23(11):2175-2190

Chung HY, Lee T-H, Kim Y-K, Kim JS (2017a) Complete chloroplast genome sequences of Wonwhang (Pyrus pyrifolia) and its phylogenetic analysis. Mitochondrial DNA Part B 2(1):325-326

Chung HY, Won SY, Kang S-H, Sohn S-H, Kim JS (2017b) The complete mitochondrial genome of Wonwhang (Pyrus pyrifolia). Mitochondrial DNA Part B 2(2):902-903

Daniell H, Lin C-S, Yu M, Chang W-J (2016) Chloroplast genomes: diversity, evolution, and applications in genetic engineering. Genome Biol 17(1):134

Davey JW, Hohenlohe PA, Etter PD, Boone JQ, Catchen JM, Blaxter ML (2011) Genome-wide genetic marker discovery and genotyping using next-generation sequencing. Nat Rev Genet 12(7):499-510

Dong W, Liu J, Yu J, Wang L, Zhou S (2012) Highly variable chloroplast markers for evaluating plant phylogeny at low taxonomic levels and for DNA barcoding. PLoS One 7(4):e35071

Dong W, Liu H, Xu C, Zuo Y, Chen Z, Zhou S (2014) A chloroplast genomic strategy for designing taxon specific DNA mini-barcodes: a case study on ginsengs. BMC Genet 15(1):138

Dong W, Xu C, Li C, Sun J, Zuo Y, Shi S, Cheng T, Guo J, Zhou S (2015) $y c f 1$, the most promising plastid DNA barcode of land plants. Sci Rep 5:8348

Group CPW, Hollingsworth PM, Forrest LL, Spouge JL, Hajibabaei M, Ratnasingham S, van der Bank M, Chase MW, Cowan RS, Erickson DL (2009) A DNA barcode for land plants. Proc Natl Acad Sci 106(31):12794-12797

Hong S-Y, Cheon K-S, Yoo K-O, Lee H-O, Cho K-S, Suh J-T, Kim S-J, Nam J-H, Sohn H-B, Kim Y-H (2017) Complete chloroplast genome sequences and comparative analysis of Chenopodium quinoa and C. album. Front Plant Sci 8:1696

Iketani H, Katayama H, Uematsu C, Mase N, Sato Y, Yamamoto T (2012) Genetic structure of East Asian cultivated pears (Pyrus spp.) and their reclassification in accordance with the nomenclature of cultivated plants. Plant Syst Evol 298(9):1689-1700

Jiang S, Zheng X, Yu P, Yue X, Ahmed M, Cai D, Teng Y (2016) Primitive genepools of Asian pears and their complex hybrid origins inferred from fluorescent sequence-specific amplification polymorphism (SSAP) markers based on LTR retrotransposons. PLoS One 11(2):e0149192

Joh HJ, Kim N-H, Jayakodi M, Jang W, Park JY, Kim YC, In J-G, Yang T-J (2017) Authentication of golden-berry P. ginseng cultivar 'Gumpoong' from a landrace 'Hwangsook' based on pooling method using chloroplast-derived markers. Plant Breed Biotechnol 5(1):16-24

Kim Y-K (2016) Diversity of Pyrus germplasm and breeding of red skin colored and scab resistant pear by using interspecific hybridization. Thesis, Chonnam National University, Jeollanam-do 
Kim H-T, Nou I-S (2016) Confirmation of parentage of the pear cultivar 'Niitaka' (Pyrus pyrifolia) based on self-incompatibility haplotypes and genotyping with SSR markers. Korean J Hortic Sci Technol 34(3):453-460

Kumar S, Stecher G, Tamura K (2016) MEGA7: molecular evolutionary genetics analysis version 7.0 for bigger datasets. Mol Biol Evol 33(7):1870-1874

Lee J, Chon J, Lim J, Kim E-K, Nah G (2017) Characterization of complete chloroplast genome of Allium victorialis and its application for barcode markers. Plant Breed Biotechnol 5(3):221-227

Ohyama K, Fukuzawa H, Kohchi T, Shirai H, Sano T, Sano S, Umesono K, Shiki Y, Takeuchi M, Chang Z (1986) Chloroplast gene organization deduced from complete sequence of liverwort Marchantia polymorpha chloroplast DNA. Nature 322(6079):572-574

Qiao J, Cai M, Yan G, Wang N, Li F, Chen B, Gao G, Xu K, Li J, Wu X (2016) High-throughput multiplex cpDNA resequencing clarifies the genetic diversity and genetic relationships among Brassica napus, Brassica rapa and Brassica oleracea. Plant Biotechnol J 14(1):409-418

Rodríguez-Ezpeleta N, Brinkmann H, Burey SC, Roure B, Burger G, Löffelhardt W, Bohnert HJ, Philippe H, Lang BF (2005) Monophyly of primary photosynthetic eukaryotes: green plants, red algae, and glaucophytes. Curr Biol 15(14):1325-1330
Rubtsov G (1944) Geographical distribution of the genus Pyrus and trends and factors in its evolution. Am Nat 78(777):358-366

Shinozaki K, Ohme M, Tanaka M, Wakasugi T, Hayashida N, Matsubayashi T, Zaita N, Chunwongse J, Obokata J, Yamaguchi-Shinozaki K (1986) The complete nucleotide sequence of the tobacco chloroplast genome: its gene organization and expression. EMBO J 5(9):2043

Sohn H-B, Kim S-J, Hwang T-Y, Park H-M, Lee Y-Y, Markkandan K, Lee D, Lee S, Hong S-Y, Song Y-H (2017) Barcode system for genetic identification of soybean [Glycine max (L.) Merrill] cultivars using InDel markers specific to dense variation blocks. Front Plant Sci 8:520

Terakami S, Matsumura Y, Kurita K, Kanamori H, Katayose Y, Yamamoto T, Katayama H (2012) Complete sequence of the chloroplast genome from pear (Pyrus pyrifolia): genome structure and comparative analysis. Tree Genet Genomes 8(4):841-854

Wicke S, Schneeweiss GM, Müller KF, Quandt D (2011) The evolution of the plastid chromosome in land plants: gene content, gene order, gene function. Plant Mol Biol 76(3-5):273-297

Yeo DY, Reed BM (1995) Micropropagation of three Pyrus rootstocks. HortScience 30(3):620-623

Zhang Q (2010) Why does biparental plastid inheritance revive in angiosperms? J Plant Res 123(2):201-206 